\title{
Highly accurate and efficient algorithm for electrostatic interaction of charged particles confined by metallic parallel plates
}

\author{
Samare Rostami, S. Alireza Ghasemi* and Ehsan Nedaaee Oskoee \\ 1 Institute for Advanced Studies in Basic Sciences, \\ P.O. Box 45195-1159, Zanjan, Iran*
}

(Dated: March 23, 2018)

\begin{abstract}
We present an accurate and efficient algorithm to calculate the electrostatic interaction of charged point particles with partially periodic boundary conditions that are confined along the nonperiodic direction by two metallic parallel plates. The method preserves the original boundary conditions and hence, it does not introduce any kind of artifacts. In addition, it enjoys the quasilinear complexity of $\mathcal{O}(N \ln (N))$, where $N$ being the number of particles in the simulation box. In fact, based on the superposition principle in electrostatics, the problem is split into two electrostatic problems where each one can be calculated by the appropriate Poisson solver. In this paper we apply the method to sodium chloride ultrathin films and investigate its dielectric response with respect to external bias voltage. We show how accurately in this method one can obtain the total charge induced on metallic boundaries to an arbitrary precision.
\end{abstract}




\section{INTRODUCTION}

In many experiments in physics and chemistry, an ionic material with slablike geometry is sandwiched between two metallic parallel plates with electric potential difference. This situation occurs in solid oxide fuel cells and batteries where an electrolyte lies between two electrodes as well as dielectric materials in capacitors. Atomistic simulations are of great importance to improve the understanding of the atomic scale mechanisms and processes in ionic materials used as an electrolyte in fuel cells or dielectric in capacitors. Electrostatic interaction in ionic materials dominates among interatomic forces and its proper calculation is thus very important for the simulations of such materials. In atomistic simulation of electrolytes, the two electrodes are usually neglected since computational methods known for Coulombic interaction do not account for the effects of the electrodes. Materials with slablike geometries are modelled with periodic boundary conditions for lateral directions and free boundary condition for the perpendicular direction. This type of boundary conditions is typically called surface, slab, or $2 \mathrm{D}+\mathrm{h}$ boundary conditions. The electrostatic interaction between charged particles and the two electrodes are typically modelled by an external electric field in the nonperiodic direction. Such a modelling does not provide information on various physical properties such as the charge density induced at the electrodes and the microscopic response of particles in the vicinity of the interface between the electrolyte and the electrodes. In addition, the effect of a uniform external electric field may be different from the electrostatic interaction in the electrolyte with the presence of the two electrodes, in particular when the electrolyte is ultrathin. In addition, there may be nonlinear phenomena that cannot be investigated by using a uniform electric field.

Due to the long ranged nature of the Coulomb potential, direct summation for free boundary conditions scales as $\mathcal{O}\left(N^{2}\right)$, where $N$ is the number of particles in the simulation box.

Several methods ${ }^{12}$ have been developed for the calculation of Coulomb interaction with free boundary conditions that have complexity of $\mathcal{O}(N \ln (N))$ while preserving the original boundary conditions. For periodic boundary conditions, particles in the periodic images must be considered as well. The Ewald method ${ }^{3}$ for fully periodic boundary conditions solves the problem due to the inclusion of particles in periodic images in the calculations, however, it has time complexity of $\mathcal{O}\left(N^{2}\right)$ and by choosing optimal parameters it can be improved to $\mathcal{O}\left(N^{\frac{3}{2}}\right)$ scaling. The scaling can be enhanced further to an $\mathcal{O}(N \ln (N))$ if fast Fourier 
transformation is employed. Several methods are developed for Coulombic interaction with slab boundary conditions. Some of these methods are in the spirit of Ewald methods ${ }^{4}[12$ and the others ${ }^{13}$ are based on the MMM ${ }^{14}$ method. The MMM method for slab boundary conditions is called MMM2D $\frac{13}{13}$ and it has the advantage that it preserves the original boundary conditions, however, its complexity is $\mathcal{O}\left(N^{\frac{5}{3}}\right)$. The standard implementation of the Ewald method based on fast Fourier transformation have been used in atomistic simulation of charged particles in slablike geometries. The major problem of the standard Ewald method for slab boundary conditions is due to the assumption that boundary conditions in all directions are considered as periodic. Therefore, in the nonperiodic direction an empty space must be used to decouple the interaction between the particles in the simulation box and those of periodic images. This approach not only makes the method inefficient but also the artificial interaction does not vanish fast enough with the size of the empty space if the system has dipole moment. Methods such particle particle particle mesh with layer correction $^{516}$ (P3MLC) have been proposed to correct for dipole-dipole interaction between periodic images. In 2007, a new method was introduced by Ghasemi and coworkers that was called particle particle particle density $\left(\mathrm{P}^{3} \mathrm{D}\right)$ method ${ }^{4}$. The $\mathrm{P}^{3} \mathrm{D}$ method is in the spirit of Ewald method and it employs plane waves for the periodic directions and finite element for the nonperiodic direction to expand the potential function and the charge density. The $\mathrm{P}^{3} \mathrm{D}$ method is the only method which not only preserves the original slab boundary conditions but also it has desirable scaling of $\mathcal{O}(N \ln (N))$.

None of the aforementioned methods in their original form can be directly employed for the electrostatic calculation of charged particles confined by metallic boundaries. In recent years, two methods were developed by Holm ${ }^{1516]}$ and coworkers that compute electrostatic interaction of charged particles confined between two dielectric materials. The image charge MMM2D ${ }^{15}($ ICMMM2D) is based on the method of image charge in electrostatics and the MMM2D method. Even though it was originally developed for Coulombic interaction of charged particles inside a dielectric material trapped between two dielectric materials, it can be applied to electrostatic interaction of charged particles confined by metallic plates. In ICMMM2D, the number of image charge particles strongly depends on the difference between dielectric constants of the electrolyte and those of surrounding materials. The computational cost of including image charge particles scales linearly with the number of particles in the simulation box. However, for large number of particles the original scaling of 
MMM2D is preserved. In principle, the method can also be applied for Coulombic interaction of charged particles between metallic plates. Based on induced charge computation ${ }^{17}$ (ICC) method, Tyagi ${ }^{16}$ and coworkers introduced a generic method that can be used for dielectric interface of arbitrary shape. The method requires a Poisson solver suitable for the same geometry in the absence of the dielectric interface. The method is called ICC ${ }^{* 16}$ in which the star stands as a representative for any Poisson solver one employs. The ICC* algorithm scales basically like the Poisson solver employed in the method. In 2013 Takae and Onuki $\frac{\sqrt{18}}{10}$ introduced a method for electrostatic interaction of charged and polar particles between metallic plates. The method is an extension of the Ewald method which accounts for the effect of image particles.

In this paper, we present a method to calculate electrostatic interaction of charged point particles between two parallel metallic plates. The method is based on superposition principle in electrostatics. In fact, similiar approaches can be applied to electrostatic interaction of charged point particles or continuous charge densities trapped by metallic boundaries with arbitrary shape. In this paper, we focus on slab geometry and we show the particular advantage of the method for this type of geometry. The method scales basically like the Poisson solver of the problem in the absence of the metallic plates. For slab geometry we employ the $\mathrm{P}^{3} \mathrm{D}$ method ${ }^{4}$ and the algorithm scales as $\mathcal{O}(N \ln (N))$. Furthermore, the method is accurate and the error in the electrostic energy and other physical quantities of interest such as total charge induced on one of the electrodes can be reduced to an arbitray value.

\section{METHOD}

\section{A. Splitting the electrostatic problem based on superposition principle}

Consider a system of $N$ particles with charges $q_{i}$ at positions $\mathbf{r}_{i}$ in an overall neutral and rectangular simulation box of dimensions $L_{x}, L_{y}$, and $L_{z}$ with periodic boundary conditions in $x$ and $y$ directions. In order to model the electrodes, boundary conditions at $z=0$ and $z=L_{z}$ are at constant voltages at $V_{l p}$ and $V_{u p}$, respectively. In fact, $\Delta V=V_{u p}-V_{l p}$ indicates the potential different between the two electrodes. The electrostatic interaction of charged particles with such boundary conditions is not Coulombic because $\frac{1}{r}$ function does not satisfy boundary conditions at $z=0$ and $z=L_{z}$. The electric potential must fulfil the 

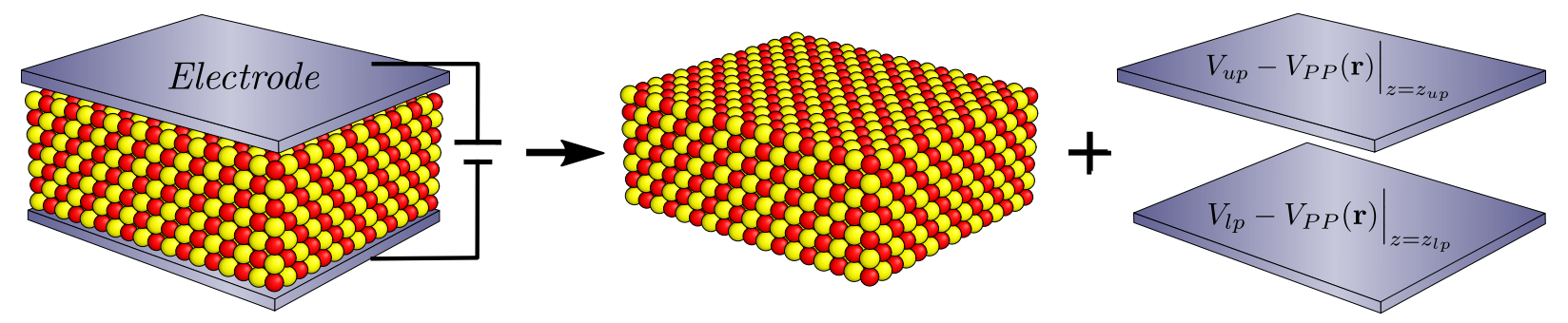

FIG. 1. (color online) Schematic illustration of the method where the electrostatic interaction of charged point particles confined by metallic parallel plates (left) is split into two parts; a system of charged point particles with free boundary condition in the $z$ direction and periodic in $x$ and $y$ directions, (middle) and two metallic parallel plates with boundary conditions given in Eq. (2) (right).

Poisson's equation,

$$
\nabla^{2} V(\mathbf{r})=-4 \pi \rho(\mathbf{r})
$$

where $\rho(\mathbf{r})$ is the charge density of point particles. The Green function of Poisson's equation for slab geometry with metallic boundary conditions is complicated and no compact functional form of the Green function is known.

We propose a method based on the superposition principle in electrostatics that can be used for the calculation of electrostatic interaction of charged particles or continuous charge densities surrounded with metallic materials. The superposition principle in electrostatics, which is due to the fact that Poisson's equation is a linear differential equation, allows us to split the problem into two problems. The corresponding electric potentials of the two problems, called $V_{1}(\mathbf{r})$ and $V_{2}(\mathbf{r})$, fulfil Poisson's equation with different charge densities, $\rho_{1}(\mathbf{r})$ and $\rho_{2}(\mathbf{r})$, respectively. Boundary conditions of the two problems are different as well. Both the new charge densities and boundary conditions are arbitrary and can be anything provided $\rho(\mathbf{r})=\rho_{1}(\mathbf{r})+\rho_{2}(\mathbf{r})$ and the two boundary conditions sum up to the original boundary conditions. Here we choose $\rho_{1}(\mathbf{r})=\rho(\mathbf{r})$ and $\rho_{2}(\mathbf{r})=0$ while for slab geometries the boundary conditions of the first problem is taken to be free in $z$ direction and periodic in $x$ and $y$ directions. In this way one can employ any Poisson solver for slablike geometry that have been summarized in Sec. If. From now on $V_{1}(\mathbf{r})$ and $V_{2}(\mathbf{r})$ are replaced by $V_{p p}(\mathbf{r})$ and $V_{s}(\mathbf{r})$, respectively, to emphasize that the first problem is the electric potential due to charged point particles and the second problem is a smooth potential which does not diverge any 
point within the simulation box. Poisson solver of the first problem must provide potential values at boundaries. Once $V_{p p}(\mathbf{r})$ is calculated we can solve the Laplace equation of the second problem with boundary conditions as

$$
\left.\left(V(\mathbf{r})-V_{p p}(\mathbf{r})\right)\right|_{\text {boundaries }}
$$

In our calculations for the Coulombic interaction in the first problem, we employ the $\mathrm{P}^{3} \mathrm{D}$ method which can provide potential values at any point in the simulation box while it is both efficient and accurate. It is also possible to use the method developed by Genovese ${ }^{19}$ and coworkers to solve the first problem. In the next section we reiterate a summary of the $\mathrm{P}^{3} \mathrm{D}$ method. The Laplace equation of the second problem is solved in a similar approach used in the $\mathrm{P}^{3} \mathrm{D}$ method. For slablike geometry, Eq. (2) is applied only to boundary conditions in the nonperiodic direction, i.e. $z=0$ and $z=L_{z}$. Fig. 1 shows a schematic view of the method presented in this section.

\section{B. P3d Method}

Consider a charge neutral set of $N$ charged point particles with charges $q_{i}$ located at position $\mathbf{r}_{i}$ in a $L_{x} \times L_{y} \times L_{z}$ simulation box. Suppose that the particles are subjected to periodic boundary conditions in two directions and free boundary conditions in the third (here we let $z$ to be the nonperiodic direction). The total electric potential energy of this systems is given by

$$
E=\frac{1}{2} \sum_{\mathbf{n}}^{\prime} \sum_{i, j=1}^{N} \frac{q_{i} q_{j}}{\left|\mathbf{r}_{i j}+\mathbf{n}\right|}
$$

where $\mathbf{r}_{i j}=\mathbf{r}_{i}-\mathbf{r}_{j}$ and $\mathbf{n}=\left(n_{x} L_{x}, n_{y} L_{y}, 0\right)$ with $n_{x}$ and $n_{y}$ being integers. The prime on the outer sum denotes that for $\mathbf{n}=0$, the term $i=j$ has to be excluded. Ewald ${ }^{3}$ showed that Eq. (3) can be split into two parts which one of them decays rapidly in real space and the other is a smooth charge density which can be treated in Fourier space very efficiently. 
Following Ewald's approach we get,

$$
\begin{aligned}
E & =\frac{1}{2} \sum_{\mathbf{n}}^{\prime} \sum_{i, j=1}^{N}\left[\frac{q_{i} q_{j}}{\left|\mathbf{r}_{i j}+\mathbf{n}\right|}-\iint \frac{\rho_{i}(\mathbf{r}) \rho_{j}\left(\mathbf{r}^{\prime}+\mathbf{n}\right)}{\left|\mathbf{r}-\mathbf{r}^{\prime}\right|} d \mathbf{r} d \mathbf{r}^{\prime}\right] \\
& +\frac{1}{2} \sum_{\mathbf{n}} \sum_{i, j=1}^{N} \iint \frac{\rho_{i}(\mathbf{r}) \rho_{j}\left(\mathbf{r}^{\prime}+\mathbf{n}\right)}{\left|\mathbf{r}-\mathbf{r}^{\prime}\right|} d \mathbf{r} d \mathbf{r}^{\prime} \\
& -\frac{1}{2} \sum_{i=1}^{N} \iint \frac{\rho_{i}(\mathbf{r}) \rho_{i}\left(\mathbf{r}^{\prime}\right)}{\left|\mathbf{r}-\mathbf{r}^{\prime}\right|} d \mathbf{r} d \mathbf{r}^{\prime}
\end{aligned}
$$

where $\rho_{i}(r)$ is a smooth spherical charge densities centered on the particle positions. The standard choice in Ewald method for the smooth atomic charge density is a Gaussian function,

$$
\rho_{i}(\mathbf{r})=\frac{q_{i}}{\left(\alpha^{2} \pi\right)^{\frac{3}{2}}} \quad \exp \left[-\frac{\left|\mathbf{r}-\mathbf{r}_{i}\right|^{2}}{\alpha^{2}}\right]
$$

Therefore, Eq. (4) can be rewritten as

$$
E=E_{\text {short }}+E_{\text {long }}-E_{\text {self }}
$$

where

$$
\begin{aligned}
E_{\text {short }} & =\frac{1}{2} \sum_{\mathbf{n}}^{\prime} \sum_{i, j=1}^{N} \frac{q_{i} q_{j} \operatorname{erfc}\left[\frac{\left|\mathbf{r}_{\mathbf{i j}}+\mathbf{n}\right|}{\alpha \sqrt{2}}\right]}{\left|\mathbf{r}_{i j}+\mathbf{n}\right|} \\
E_{\text {long }} & =\frac{1}{2} \sum_{\mathbf{n}} \sum_{i, j=1}^{N} \iint \frac{\rho_{i}(\mathbf{r}) \rho_{j}\left(\mathbf{r}^{\prime}+\mathbf{n}\right)}{\left|\mathbf{r}-\mathbf{r}^{\prime}\right|} d \mathbf{r} d \mathbf{r}^{\prime} \\
E_{\text {self }} & =\frac{1}{\alpha \sqrt{2 \pi}} \sum_{i=1}^{N} q_{i}^{2} .
\end{aligned}
$$

Complementary error function in Eq. (6a) decays exponentially. It can thus be made of finite range by introducing a cutoff. Therefore, the calculation of the first term can be done with linear scaling.

The system is considered to have a nonzero charge density just within $\left[z_{l b}, z_{u b}\right]$ for nonperiodic direction. The simulation cell in periodic directions are the same size as the original cell and in z direction, $z_{u b}-z_{l b}$ is $L_{z}$ plus twice the cutoff for Gaussian charge density. Hence we can write the domain as,

$$
\mathcal{V}:=\left[0, L_{x}\right] \otimes\left[0, L_{y}\right] \otimes\left[z_{l b}, z_{u b}\right]
$$


$E_{\text {long }}$ is the electrostatic energy of charge density comprised of superposition of atomic Gaussian functions. The electric potential due to atomic Gaussian functions, $V_{G F}(r)$, can be obtained by solving Poisson's equation,

$$
\nabla^{2} V_{G F}(\mathbf{r})=-4 \pi \rho(\mathbf{r})
$$

where $\rho(\mathbf{r})=\sum_{i=1}^{N} \rho_{i}(\mathbf{r})$. Eq. (8) is solved for simulation box given in Eq. (7) with periodic boundary conditions in $x$ and $y$ directions. We expand the potential function and the charge density in terms of Fourier series,

$$
\begin{array}{rlrl}
V_{G F}(x, y, z) & = & & \sum_{k, l=-\infty}^{\infty} c_{k l}(z) \exp \left[2 i \pi\left(\frac{k x}{L_{x}}+\frac{l y}{L_{y}}\right)\right], \\
\rho(x, y, z) & = & \sum_{k, l=-\infty}^{\infty} \frac{\eta_{k l}(z)}{-4 \pi} \exp \left[2 i \pi\left(\frac{k x}{L_{x}}+\frac{l y}{L_{y}}\right)\right] .
\end{array}
$$

Inserting Eqs. (9a) and 9b) in Eq. (8) yields,

$$
\left(\frac{d^{2}}{d z^{2}}-g_{k l}^{2}\right) c_{k l}(z)=\eta_{k l}(z)
$$

where

$$
g_{k l}:=2 \pi \sqrt{\frac{k^{2}}{L_{x}^{2}}+\frac{l^{2}}{L_{y}^{2}}}
$$

In order to calculate Fourier coefficients $c_{k l}(z)$ in Eq. (10) one needs to determine boundary conditions at $z \rightarrow \pm \infty$. As it is explained in Ref. [4], we have $V(x, y, z \rightarrow \pm \infty)=\mp \beta$ where $\beta$ is proportional to the dipole moment of the charge distribution along the $\mathrm{z}$ direction,

$$
\beta=\frac{1}{2} \int_{z_{l b}}^{z_{u b}} \eta_{00}\left(z^{\prime}\right) z^{\prime} d z^{\prime}
$$

To deal with the Eq. (10) with above boundary conditions, one can write the following conditions for the $g$ 's :

- $g_{00}=0 \Rightarrow \frac{d^{2}}{d z^{2}} c_{00}(z)=\eta_{00}(z)$, we solve this differential equation with boundary condition $c_{00}(z \rightarrow \pm \infty)=\mp \beta$

- $g_{k l} \neq 0 \Rightarrow\left(\frac{d^{2}}{d z^{2}}-g_{k l}^{2}\right) c_{k l}(z)=\eta_{k l}(z)$, for all of these differential equations we have to impose boundary conditions of the form $c_{k l}(z \rightarrow \pm \infty)=0$. 
$c_{00}(z)$ is constant for $z \notin\left[z_{l b}, z_{u b}\right]$ and therefore, one has to impose the Dirichlet boundary conditions $c_{00}\left(z_{l b}\right)=\beta$ and $c_{00}\left(z_{u b}\right)=-\beta$. It is explained in Ref. [4] that all differential equations with $|k|+|l|>0$ must be solved subject to Robin boundary conditions given by the following equations at $z_{l}$

$$
c^{\prime}\left(z_{l b}\right)-g_{k l} c\left(z_{l b}\right)=0
$$

and at $z_{u b}$

$$
c^{\prime}\left(z_{u b}\right)+g_{k l} c\left(z_{u b}\right)=0 .
$$

To solve the differential equations in Eq. (10) with boundary conditions explained above, finite element method are employed as explained in Ref. [4] and its appendix. Once $c_{k l}(z)$ are

obtained, the potential function is calculated using a reverse Fourier transform. Then the electrostatic energy $E_{l o n g}$ and atomic forces can be calculated. The $\mathrm{P}^{3} \mathrm{D}$ method provides values of the potential function at upper and lower planes that are necessary to build the boundary conditions of the second problem.

\section{Laplace's equation of the second problem}

As it is explained in sec. IIA, we should calculated values of the potential function of the first problem at boundaries. The potential function of the $\mathrm{P}^{3} \mathrm{D}$ method is given by

$$
V_{p p}(\mathbf{r})=V_{G F}(\mathbf{r})+\sum_{\mathbf{n}} \sum_{i=1}^{N} \frac{q_{i} \operatorname{erfc}\left[\frac{\left|\mathbf{r}_{\mathrm{i}}-\mathbf{r}+\mathbf{n}\right|}{\alpha}\right]}{\left|\mathbf{r}_{i}-\mathbf{r}+\mathbf{n}\right|} .
$$

The first term in Eq. (15) is available on the grid points. If the grid points are chosen such that the upper and lower boundaries lie on the grid points, the first term can be calculated on boundaries at no cost and without interpolation, as it is done in our implementation. The second term in Eq. (15) decays exponentially and it can be made of finite range by introducing a cutoff. The Laplace equation must be solved subject to the following boundary conditions for the upper plane

$$
V_{b u p}(x, y)=V_{u p}-\left.V_{p p}(\mathbf{r})\right|_{z_{u p}},
$$

and for the lower plane

$$
V_{b l p}(x, y)=V_{l p}-\left.V_{p p}(\mathbf{r})\right|_{z_{l p}},
$$


where in our implementation $z$ coordinate of lower and upper planes, $z_{l p}$ and $z_{u p}$, are zero and $L_{z}$, respectively. In a similar approach to sec. IIB, we expand the potential function in terms of Fourier series for $x$ and $y$ directions with expansion coefficients of $f_{k l}(z)$. Replacing the Fourier series expansion in the Laplace equation, it yields

$$
\left(\frac{d^{2}}{d z^{2}}-g_{k l}^{2}\right) f_{k l}(z)=0
$$

where $g_{k l}$ is given by Eq. (11). Eq. (18) differs from Eq. 10 in two aspects, first it is homogeneous and second its boundary conditions is different. In order to obtain boundary conditions of Eq. (18), $V_{b u p}(x, y)$ and $V_{b l p}(x, y)$ are expanded in term of Fourier series for $x$ and $y$ directions with expansion coefficients of $a_{k l}$ and $b_{k l}$, respectively. Eq. (18) can be solved analytically and there is no need to employ the finite element method used for solving Eq. (10). $f_{00}(z)$ is a linear function given by

$$
f_{00}(z)=\frac{\left(a_{00}-b_{00}\right) z+\left(b_{00} z_{u p}-a_{00} z_{l p}\right)}{z_{u p}-z_{l p}},
$$

and for $f_{k l}(z)$ with $|k|+|l|>0$, we have

$$
f_{k l}(z)=\frac{a_{k l} \sinh \left(g_{k l}\left(z-z_{l p}\right)\right)+b_{k l} \sinh \left(g_{k l}\left(z_{u p}-z\right)\right)}{\sinh \left(g_{k l}\left(z_{u p}-z_{l p}\right)\right)} .
$$

Using Eqs. (19) and (20), $f_{k l}(z)$ are calculated on the grid points and subsequently $V_{s}(\mathbf{r})$ are obtained on the grid points by performing a reverse Fourier transformation. The electrostatic energy contribution of the second problem is given by

$$
E_{s}=\sum_{i=1}^{N} q_{i} V\left(\mathbf{r}_{i}\right)
$$

The potential function, $V_{s}(\mathbf{r})$, is not known at atomic positions. Therefore, we use Lagrange polynomial of order eight to interpolate the potential function at atomic positions. The total electrostatic energy is the sum of the four terms, three of which are due to the electrostatic energy of point particles given by Eqs. (6a), (6b), (6c) and the fourth term is the electrostatic energy of the smooth potential given by Eq. (21).

\section{NUMERICAL RESULTS}

We illustrate the accuracy of the method for two important quantities relevant to electrostatic problems of point particles confined by metallic boundaries. A fast and robust 


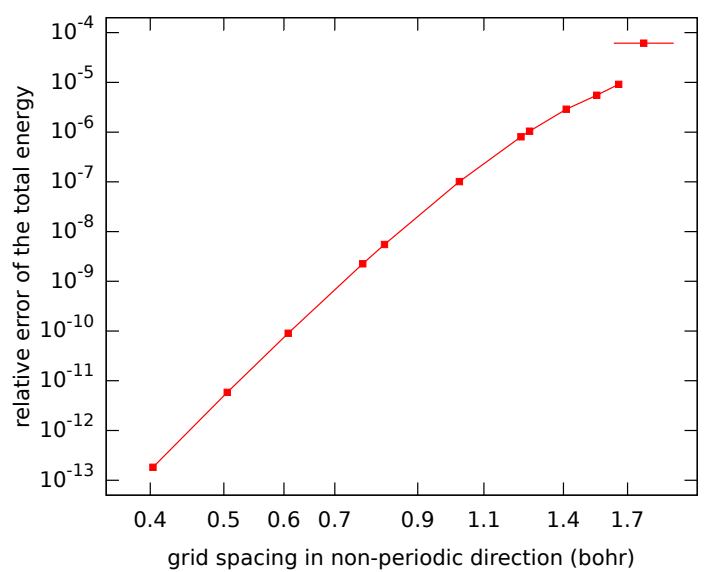

FIG. 2. (color online) Relative error in the total energy versus grid spacing in $z$ direction. On this double logarithmic plot, the curve has an asymptotic slope of 14 and machine precision can be reached.

convergence in electrostatic energy is vital for an algorithm for solving Poisson's equation. Due to the use of Fourier series in periodic directions, exponential convergence rate is observed with respect to grid spacing in those directions. However, the convergence rate in the nonperiodic direction, due to the use of polynomial as the basis set, is algebraic $\mathcal{O}\left(h_{z}^{2 m}\right)$ where $m$ is the degree of the polynomial used in the finite element method. Fig. 2 illustrates the convergence rate in total energy in terms of grid spacing in the nonperiodic direction. Detailed explanation of the finite element approach employed in this work is given in Ref. [4]. Fig. 2 shows that the error in total energy can be reduced down to machine precision. Another interesting quantity in electrostatic problems with metallic boundaries is the surface charge density induced on boundaries. In order to investigate the accuracy of the method for the surface charge density, we assess the total charge induced on the boundaries. Due to translational invariance of the system in $x$ and $y$ directions, the total charge induced on upper and lower planes must remain constant under translation of particles along these directions. In fact, the total charge oscillates due to the discretization of equations. Fig. 3 shows the oscillation amplitude of the total charge induced on planes with respect to the translation in periodic directions for two values of grid spacings in the periodic directions. The deviation from constant value is very small. Furthermore, the oscillation amplitude of the total charge decays rapidly by more than five orders of magnitude as the grid spacing is reduced by about 30 percent. 


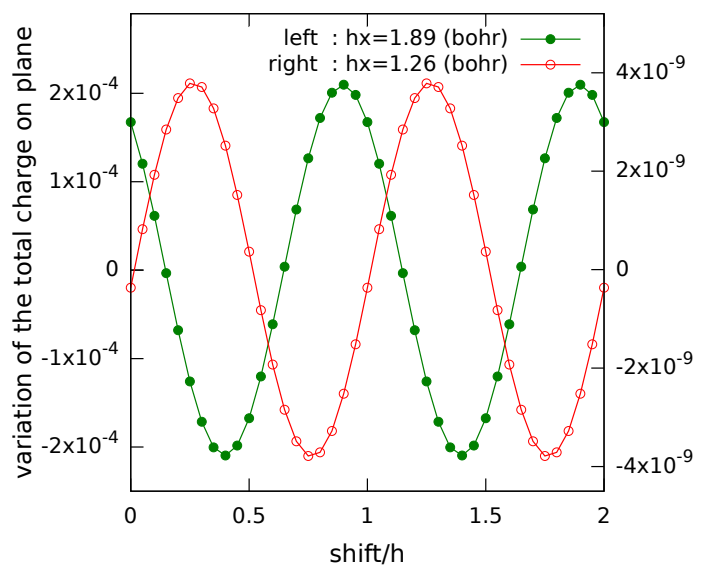

FIG. 3. (color online) Total induced charge on metallic plates versus shift of charged particles in a periodic direction for two different values of grid spacing.

We performed zero temperature atomistic simulation of sodium chloride system containing 1000 particles using structural relaxation by means of energy minimization. The short range interactions are obtained from the Born-Mayer-Huggins-Fumi-Tosi ${ }^{20}$ (BMHFT) rigid-ion potential, with the parameters given in Ref.[21]. Fig. 4 illustrates the total charge induced on the upper plane during minimization process of several structures with initial configurations chosen to be randomly displaced particles from equilibrium. As it is shown in Fig. 4, the final configurations have the same total charge induced on upper/lower planes even though the trajectories are different and induced charges are different during various trajectories. Obviously, the total charge induced on the upper plane for the final configuration in a minimization process could be different from others if the final configuration would be different from those of other minimization processes. This could happen if the random displacement of the initial structures were too large.

The response of insulators and semiconductors to external electric field is of great interest both from experimental and theoretical aspects. The typical approach to study external electric field is to apply an external uniform electric field, however, in experimental setup such electric field is caused by voltage difference of two electrodes surrounding the material. In the uniform electric field approach one cannot study nonlinear response of the material to the external electric field. This is of great significance for ultrathin films. Furthermore, in practical situations ions do not feel a homogeneous electric field and thus ionic displacements are indeed nonuniform. Ions in the vicinity of the interface of the material and 


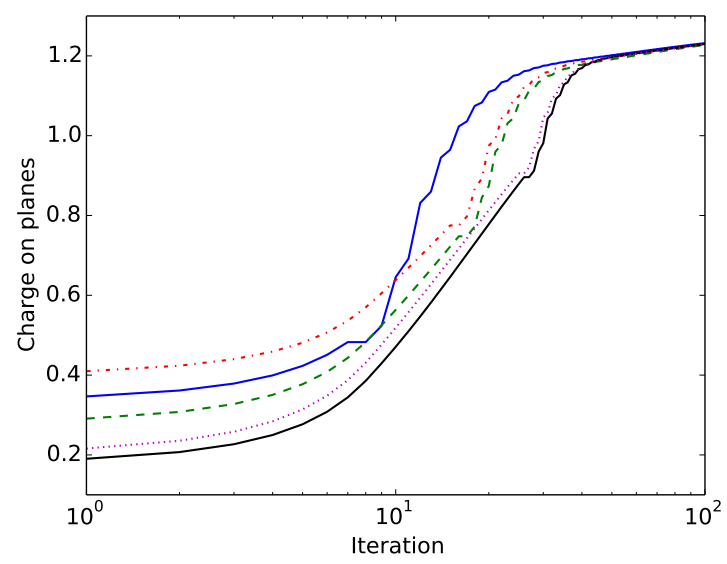

FIG. 4. (color online) Total charge induced on the metallic plates versus iteration during minimization process. Each curve represents different sodium chloride initial configuration in which particles are slightly randomly displaced from the rock salt structure. The final value of induced charge is independent of the trajectory since the final structure of all different initial configurtions is the same.

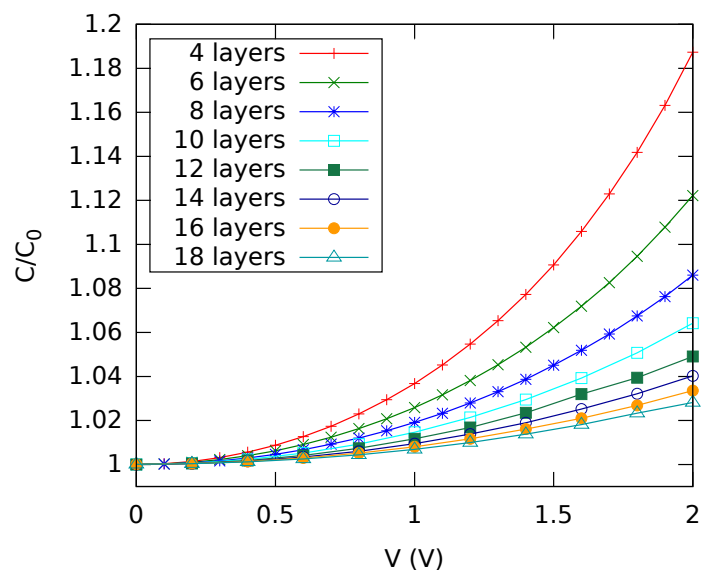

FIG. 5. (color online) Capacitance normalized to capacitance at zero bias voltage versus potential difference of the two metallic parallel plates. $\left(C_{0}=\left.\frac{d Q}{d V}\right|_{V=0}\right)$

the electrodes feel a more oscillatory electric field and studying this phenomena is possible with the approach presented in this paper. Here we study nonlinear dielectric behavior of sodium chloride as a prototype of alkali halide. The nonlinear behavior shows up in large electric fields. Here we investigate this phenomena for ultrathin $\mathrm{NaCl}$ films from 4 to 18 layers. Due to the nonlinear behavior in the response of the material to electrostatic field, the total charge induced on upper/lower plane is not a linear function of the bias voltage, 


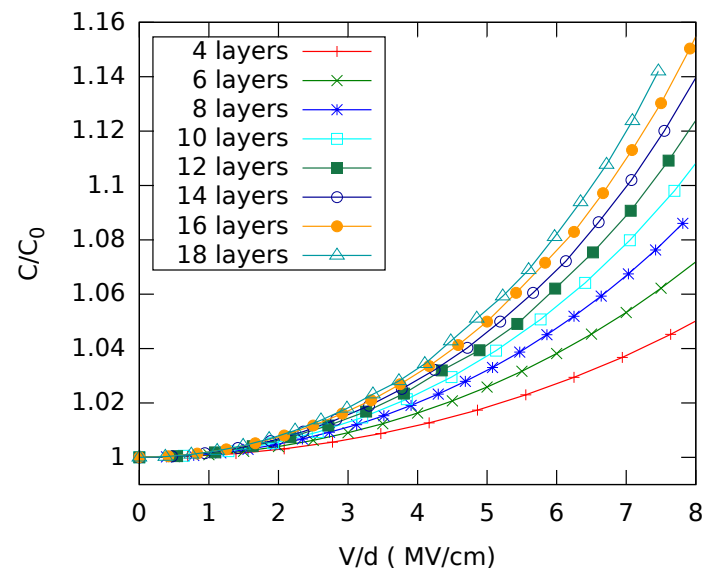

FIG. 6. (color online) Capacitance normalized to capacitance at zero bias voltage versus average external electric field due to the two metallic parallel plates.

consequently, the capacitance is not a constant and it should be calculated by

$$
C(V)=\frac{d Q(V)}{d V}
$$

Fig. 5 illustrates the ratio of capacitance to the capacitance at zero bias limit in terms of bias voltage. Fig. 5 shows that the nonlinear contribution to capacitance increases strongly as a function of bias voltage and it can be as much as $20 \%$ of the linear contribution. Based on the curves in Fig. 5, one may assume that the thiner the film the larger is the nonlinear contribution. This is in fact the opposite. The nonlinear behavior is stronger for thicker films. Fig. 6 shows the ratio of capacitance to the capacitance at zero bias limit in terms of electric field. The electric field is calculated by ratio of the potential difference between the two electrodes to their distance. From curves in Fig. 6, it is obvious that the nonlinear contribution to capacitance increases as the thickness of the film grows for a given value of electric field. However, the increase in the nonlinear contribution slows down for thick films and it is expected to approach to zero at the limit of very thick films. Electric field in the calculations shown in Fig. 6, are very large and cannot be applied to bulk $\mathrm{NaCl}$ since they are larger than electrical breakdown of $\mathrm{NaCl}$. In general electrical breakdown of thin films is larger than that of bulk and such large electric fields used in these calculations may be plausible.

We have also calculated the dielectric constant of sodium chloride films with respect to the thickness of the film. Dielectric properties of sodium chloride films have been investigated using ab initio techniques in Ref. [22], where a uniform external electric field is applied to 
TABLE I. Dielectric constant $(K)$ of various $\mathrm{NaCl}$ ultrathin films with respect to the number of layers $(n)$ in the film.

\begin{tabular}{c|cccccccc}
\hline \hline$n$ & 10 & 20 & 30 & 40 & 50 & 60 & 80 & 100 \\
\hline$K$ & 3.73 & 4.01 & 4.12 & 4.18 & 4.28 & 4.24 & 4.28 & 4.31 \\
\hline \hline
\end{tabular}

sodium chloride films. It would be very interesting if one could apply a combination of our method and ab initio techniques such as density functional theory to investage dielectric properties of ionic ultrathin films confined with metallic plates. Table I presents the list of dielectric constant for $\mathrm{NaCl}$ ultrathin films with thickness of 10 to 100 layers. The dielectric constants are obtained with relation $K=\frac{C_{0}}{C_{v a c}}$ where $C_{v a c}$ is the capacitance in the absence of the film. $C_{0}$ is the capacitance at zero bias limit and for thick films it is virtually the same as the value obtained by the ratio of the total charge induced on upper/lower plane to the potential difference of the two electrodes. A relaxation with very tight convergence criteria is required for thick films and the system becomes ill conditioned with the growth of the number of particles. Indeed, these two points prevent us to calculate the dielectric constant for very thick films. Table【indicates that the dielectric constant increases with the growth of the $\mathrm{NaCl}$ films, however, the growth slows down for thick films. It is expected that the diecltric constant approaches to a fixed value which corresponds the dielctric constant of bulk sodium chloride. In fact, the quaility of the interatomic potential is decisive for the accuracy of the dielectric constant. In Fumi-Tosi force field ions are treated with fixed charges and therefore, the dielectric constant obtained in our approach accounts only for the ionic degrees of freedom of the material and does not include the effects due to the electronic polarization.

\section{CONCLUSION}

We presented a new method for electrostatic interaction of charged point particles confined between two parallel metallic plates. Due to the linear nature of governing electrostatic equations, the electric potential is assumed to be a superposition of two parts; one singular part in the absence of metallic plates that is due to the existence of point charges in the simulation box, and the other is a smooth potential, imposed by the metallic boundaries. 
In this way, we were able to employ a previously developed method for the similar geometry without the presence of the metallic plates. The second part was solved in a similar approach but slightly different due to the resulting homogeneous ordinary differential equation that could be solved analytically. The method is very efficient and it has a quasilinear complexity of $\mathcal{O}(N \ln (N))$. In order to investigate the accuracy and efficiency of the method, zero temperature atomistic simulations of sodium chloride system were performed. The relative error of the total energy depends on the grid spacing, however, it decreases very rapidly with reducing the mesh size and it can be made sufficiently small, even zero limited by the machine precision. Furthermore, by imposing the potential difference between metallic plates and allowing the system to relax, nonlinear behavior of capacitance of sodium chloride ultrathin films was investigated. The nonlinear behavior grows as the number of layers in the film is increased, however, the growth slows down as the film thickness increases. In addition, we calculated the dielectric constant of the sodium chloride ultrathin films where it was shown that the dielectric constant increases with growth of the film. The dielectric constant of sodium chloride films with 100 layers is about $20 \%$ lower than experimental dielectric constant of bulk sodium chloride. In a forthcoming study we will employ our method and investigate finite temperature effects on dielectric properties of sodium chloride ultrathin films. 
* aghasemi@iasbs.ac.ir

1 L. Greengard and V. Rokhlin, J. Comp. Phys. 73, 325 (1987).

2 A. Neelov, S. A. Ghasemi, and S. Goedecker, J. Chem. Phys. 127, 024109 (2007).

3 P. P. Ewald, Ann. Phys. 64, 253 (1921).

4 S. A. Ghasemi, A. Neelov, and S. Goedecker, J. Chem. Phys. 127, 224102 (2007).

5 A. Arnold, J. de Joannis, and C. Holm, J. Chem. Phys. 117, 2496 (2002).

6 J. de Joannis, A. Arnold, and C. Holm, J. Chem. Phys. 117, 2503 (2002).

7 D. M. Heyes, M. Barber, , and J. H. R. Clarke, J. Chem. Soc., Faraday Trans. 2 73, 1485 (1977).

8 J. Hautman and M. L. Klein, Mol. Phys. 75, 379 (1992).

9 B. R. A. Nijboer, Physica A 125, 275 (1984).

10 E. Spohr, J. Chem. Phys. 107, 6342 (1997).

11 Y. J. Rhee, J. W. Halley, J. Hautman, and A. Rahman, Phys. Rev. B 40, 36 (1989).

12 I. C. Yeh and M. L. Berkowitz, J. Chem. Phys. 111, 3155 (1999).

13 A. Arnold and C. Holm, Comput. Phys. Commun. 148, 327 (2002).

14 R. Sperb, Mol. Simul. 20, 179 (1998).

15 S. Tyagi, A. Arnold, and C. Holm, J. Chem. Phys. 127, 154723 (2007).

16 S. Tyagi, M. S. uzen, M. Sega, M. Barbosa, S. Kantorovich, and C. Holm, J. Chem. Phys. 132, $154112(2010)$.

17 D. Boda, D. Gillespie, W. Nonner, D. Henderson, and B. Eisenberg, Phys. Rev. E 69, 046702 (2004).

18 K. Takae and A. Onuki, J. Chem. Phys. 139, 124108 (2013).

19 L. Genovese, T. Deutsch, and S. Goedecker, J. Chem. Phys. 127, 054704 (2007).

20 M. P. Tosi and F. G. Fumi, J. Phys. Chem. Solids 25, 45 (1964).

21 F. H. Ree and A. C. Holt, Phys. Rev. B 8, 826 (1973).

22 T. Ono and K. Hirose, Phys. Rev. B 72, 085105 (2005). 